\title{
O método de Gauss-Newton uma aplicação em antenas filamentares
}

\author{
Valcir J. da C. Farias Alessandra L. de Oliveira Marissol T. Sperotto \\ Programa de Pós-graduação em Matemática e Estatística-ICEN-UFPA \\ Rua Augusto Corrêa, 01, Belém, Pará \\ E-mail: valcir@ufpa.br; alima485@gmail.com; marissol@ufpa.br.
}

\begin{abstract}
Resumo - O projeto da antena Yagi-Uda foi otimizado aplicando o método de Gauss-Newton. A otimização consistiu em especificar intervalos de valores para a diretividade, razão frente-costa e ângulo de meia potência e, partindo de um modelo inicial pré-definido, foram determinados os melhores valores para o comprimento e espaçamento dos elementos. Para a modelagem direta, foi utilzado o método dos momemtos sobre a equação integral de Pocklington, a qual consistiu em obter os valore de diretividade, razão frente-costa e ângulo de meia potência a partir do comprimento e do espaçamento entre elementos conhecidos. O procedimento foi aplicado na síntese de antenas Yagi-Uda com cinco elementos e os resultados foram encontrados tão bons quanto os obtidos na literatura aplicando outros métodos de otimização.
\end{abstract}

Palavras chaves - Antena Yagi-Uda, método de Gauss-Newton, otimização.

\section{INTRODUÇÃO}

A antena Yagi-Uda foi introduzida em 1920 por S. Uda [1]. Uma antena Yagi-Uda convencional consiste em dipolos lineares paralelos dos quais somente um, normalmente o segundo elemento, é energizado por uma fonte, os demais são elementos parasitas. O primeiro elemento funciona como refletor, o qual possui tamanho maior que o elemento energizado. Do terceiro até o n-ésimo são elementos diretores e são menores que o elemento fonte. A Figura 1 mostra uma antena Yagi-Uda com seis elementos.

Apesar da aparência simples da antena Yagi-Uda, o projeto desse dispositivo não é uma tarefa fácil, principalmente por que existem muitas inter-relações entre as variáveis envolvidas no projeto, como exemplo, os elementos são eletromagneticamente acoplados e uma pequena variação no comprimento e/ou no espaçamento entre os elementos da antena pode alterar a distribuição de corrente sobre todos os componentes. As dificuldades de projeto fizeram com que a atenção de alguns pesquisadores se voltasse para a otimização da antena Yagi-Uda [2-4].

Cheng [2] usou o método do gradiente para otimizar o ganho e a impedância de entrada da antena Yagi-Uda, seus resultados aumentaram em $80 \%$ o ganho de um projeto inicial de um dispositivo Yagi-Uda.

Jones and Joines [3] e Ramos et al. [4] usaram algoritmo genético para o projeto da antena Yagi-Uda, seus resultados foram tão bons quanto os apresentados por [2].

Para problemas de otimização com poucos parâmetros os métodos de busca local, como Newton, Quasi-Newton e Gauss-Newton possuem um bom desempenho. Além disso, essas técnicas são computacionalmente, nestes casos, tão rápidas quanto os métodos de busca global, como o algoritmo genético. Dessa forma, se torna atrativo desenvolver a otimização da antena Yagi-Uda aplicando uma técnica de busca local.

Este trabalho aplica o método de Gauss-Newton para otimizar diretividade, ângulo de meia potência e razão frente-costa da antena Yagi-Uda a partir do ajuste dos valores dos comprimentos e espaçamentos entre elementos desse dispositivo de radiação.

\section{MÉTOdo de GAUSS-NEWTON}

O objetivo do projeto é desenvolver uma antena Yagi-Uda que reúna algumas características requeridas para um bom desempenho desse dispositivo. As características que serão especificadas neste trabalho são: número de elementos, sendo um refletor e um elemento energizado; diretividade; razão frente-costa; e ângulo de meia. Os valores dos tamanhos e dos espaçamentos entre os elementos serão ajustados pelo processo de otimização de GaussNewton. 
Um projeto inicial da antena será estabelecido e, a partir desses dados, aplica-se o método de Gauss-Newton até que as faixas de valores das características pré-estabelecidas sejam atingidas.

A qualidade do processo de otimização está relacionada, matematicamente, a uma função Custo.

A função custo utilizada no processo de otimização de Gauss-Newton, neste trabalho, foi a mesma desenvolvida por [5-6], e é dada pela expressão (1).

$$
C(m)=\frac{1}{2}\left\{\mu\left[\left\|\overline{\bar{W}}_{d} \cdot\left(f(m)-d^{o b s}\right)\right\|^{2}-\chi^{2}\right]+\left\|\overline{\bar{W}}_{m}\left(m-m_{r}\right)\right\|^{2}\right\}
$$

Onde $\mu(0<\mu<\infty)$ é o parâmetro de regularização (multiplicador de Lagrange); $\chi^{2}$ é um valor pré-estabelecido de ajuste dos dados; $m$ é o vetor contendo os valores que serão ajustados (parâmetros do modelo), que neste trabalho foram os tamanhos e os espaçamentos entre os elementos; $f(m)$ é o operador modelagem direta; $d^{\text {obs }}$ é o vetor contendo os valores requeridos para o projeto da antena (diretividade, razão frente-costa e ângulo de meia potência); $W_{m}$ á inversa da matriz covariância dos dados que serão ajustados; e $W_{d}$ é a inversa da matriz covariância dos dados pré-estabelecidos para o projeto.

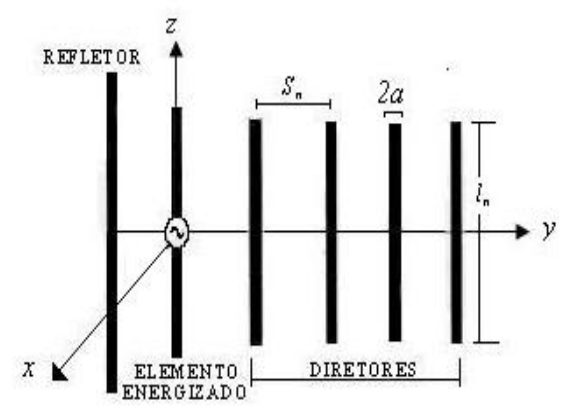

Figura 1: Antena Yagi-Uda com seis elementos.

O problema apresentado aqui é de otimização não-linear que para o qual aplica-se o método iterativo de Newton. Esta técnica é baseada em uma representação quadrática da função custo. O modelo quadrático é obtido tomando-se os três primeiros termos da expansão por série de Taylor da função custo (1) em torno da k-ésima iteração $\left(m_{k}\right)$, assim:

$$
C\left(m_{k}+\Delta m_{k}\right)=C\left(m_{k}\right)+g^{T}\left(m_{k}\right) \cdot \Delta m_{k}+\frac{1}{2} \Delta m_{k}^{T} \cdot G\left(m_{k}\right) \cdot \Delta m_{k}
$$

onde T denota matriz transposta e $\Delta m_{k}=m_{k+1}-m_{k}$ é o incremento do vetor dos parâmetros em direção ao ponto estacionário da função custo $C\left(m_{k}\right) ; \bar{g}(m)=\nabla C(m)$ é o vetor gradiente da função custo, ou seja:

$$
g(m)=\nabla C(m)=\left[g_{n} \equiv \frac{\partial C}{\partial m_{n}}, n=1,2,3, \cdots, N\right]=\mu J^{T}(m) \cdot W_{d}{ }^{T} \cdot W_{d} \cdot e(m)+W_{x}{ }^{T} \cdot W_{x} \cdot\left(m-m_{r}\right)
$$

$m_{n}$ é a n-ésima componente do vetor dos parâmetros do modelo $m ; e(m)=f(m)$ - $d^{o b s}$ é o vetor erro residual; e $J(m)$ é uma matriz cuja dimensão é $M \times N$ denominada de Jacobiano (ou sensitividade) e é dada por:

$$
J(m)=\left[\frac{\partial e_{l}(m)}{\partial m_{n}}, \quad l=1,2,3, \cdots, M ; \quad n=1,2,3, \cdots, N\right]
$$

$G(m)=\nabla \nabla C(m)$ é o Hessiano da função custo o qual é uma matriz simétrica de ordem $\mathrm{N} \times \mathrm{N}$ dada por:

$$
G(m)=\nabla \nabla C(m)=\left[G_{n l}=\frac{\partial^{2} C}{\partial m_{n} \partial m_{l}}, n, l=1,2,3, \cdots, N\right]=W_{m} \cdot W_{m}{ }^{T}+\mu\left[J^{T}(m) \cdot W_{d}{ }^{T} \cdot W_{d} \cdot J(m)+Q(m)\right]
$$

com $Q(m)=\sum_{l}^{M} f_{l}(m) F_{l}^{T}(m)$ sendo $f_{l}(m)$ o $l$-ésimo elemento do vetor $f_{l}(m)=W_{d} . e(m), \mathrm{e}$

$$
F_{l}(m)=\nabla \nabla f_{l}(m)=\left[\frac{\partial^{2} f_{l}}{\partial m_{i} \partial m_{j}} \quad i, j=1,2,3, \cdots, N\right]
$$

O mínimo de (2) é obtido quando $\Delta m_{k}$ for um mínimo da função quadrática 


$$
\phi(\Delta m)=g^{T}\left(m_{k}\right) \cdot \Delta m+\frac{1}{2} \Delta m^{T} \cdot G\left(m_{k}\right) \cdot \Delta m
$$

A função $\phi(\Delta m)$ tem um ponto estacionário (ponto crítico) em $\Delta m_{k}$ somente se o gradiente de $\phi(\Delta m)$ for para zero em $\Delta m_{k}$, isto é:

$$
\nabla \phi\left(\Delta m_{k}\right)=g^{T}\left(m_{k}\right)+G\left(m_{k}\right) \cdot \Delta m_{k}=0
$$

Assim, o ponto estacionário $\Delta m_{k}$ da função $\phi(\Delta m)$ será a solução do sistema de equações lineares:

$$
G\left(m_{k}\right) \cdot \Delta m_{k}=-g\left(m_{k}\right)
$$

Dependendo da definição da matriz Hessiano, o ponto estacionário dado por (5) pode ser um ponto de mínimo, máximo ou de sela. De acordo com [5], a condição sobre a matriz Hessiano $G(m)$ em ser singular ou não singular e sua definição (positiva, negativa definida ou indefinida) podem ser ajustada por uma escolha apropriada do parâmetro de regularização $\mu$.

No método de Gauss-Newton despreza-se as derivadas de segunda ordem da função custo com relação as componentes do vetor dos parâmetros do modelo $(m)$, ou seja, não considera-se o termo $Q$. Assim, o Hessiano, no método de Gauss-Newton, será dado por:

$$
G(m)=W_{m} \cdot W_{m}{ }^{T}+\mu J^{T}(m) \cdot W_{d}^{T} \cdot W_{d} \cdot J(m)
$$

O método reduz-se em resolver um sistema de equações lineares apresentado em (7).

$$
\left[J^{H} \cdot W_{d}{ }^{T} \cdot W_{d} J^{H}+W_{m}{ }^{T} \cdot W_{m}\right] \cdot \Delta m_{j}=\mathrm{J}^{\mathrm{H}} \cdot W_{d}{ }^{T} \cdot W_{d} \cdot\left[d^{o b s}-f\left(m_{j}\right)\right]-W_{m}{ }^{T} \cdot W_{m} \cdot m_{j}
$$

O sistema de equações lineares (7) foi resolvido aplicando o método da eliminação de Gauss. A modelagem direta, ou seja, o projeto da antena Yagi-Uda a partir das entradas de comprimento e espaçamento entre os elementos da antena e como saída a diretividade, ângulo de meia potência e razão frente-costa, é baseado no método dos momentos apresentado em [1].

\section{Resultados}

Para aplicar o procedimento descrito neste trabalho na otimização da diretividade, do ângulo de meia potência e da razão frente-costa da antena Yagi-Uda, utilizaremos o exemplo de antena com cinco elementos. Os elementos foram considerados os seguintes dados iniciais:

Comprimento do 1 diretor $=0.419 \lambda$;

Comprimento do 2 diretor $=0.437 \lambda$;

Comprimento do 3 diretor $=0.407 \lambda$;

Comprimento do refletor $=0.483 \lambda$;

Comprimento da fonte $=0.434 \lambda$;

espaçamento entre fonte e 1 diretor $=0.271 \lambda$;

espaçamento entre 1 e 2 diretor $=0.362 \lambda$;

espaçamento entre 2 e 3 diretor $=0.390 \lambda$; e

espaçamento entre fonte e refletor $=0.224 \lambda$.

Onde o diagrama de radiação dessa antena para o plano H é mostrado na Figura 2.

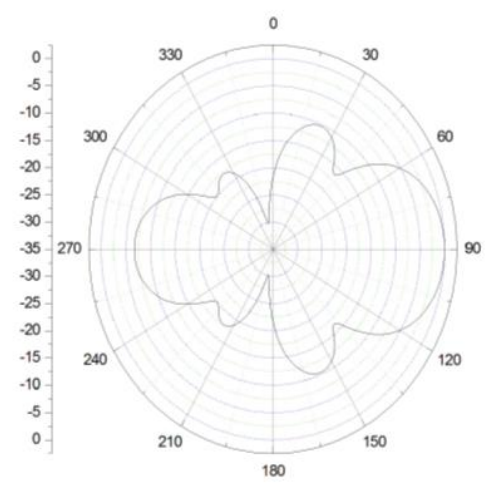

Figura 2: Diagrama de radiação (plano H) da antena Yagi-Uda de cinco elementos com os dados iniciais. 
Os parâmetros de diretividade, razão frente-costa e ângulo de meia potência, para os dados iniciais, são apresentados a seguir: Ângulo de meia potência (plano E)=43,08 graus; Ângulo de meia potência (plano $\mathrm{H}$ )=47,81 graus; Razão frente-costa $($ plano $\mathrm{E})=6,6602 \mathrm{~dB}$; Razão frente-costa $($ plano $\mathrm{H})=6,6516 \mathrm{~dB}$; Diretividade $=11,444 \mathrm{~dB}$.

Após a aplicação do método de Gauss-Newton, obteve-se os valores indicados a seguir para comprimento e espaçamento entre os elemento da antena:

Comprimento do 1 diretor $=0.4308273 \lambda$;

Comprimento do 2 diretor $=0.4308273 \lambda$;

Comprimento do 3 diretor $=0.4308273 \lambda$;

Comprimento do refletor $=0.4884964 \lambda$;

Comprimento da fonte $=0.4746618 \lambda$;

espaçamento entre fonte e 1 diretor $=0.2308273 \lambda$;

espaçamento entre 1 e 2 diretor $=0.323309 \lambda$;

espaçamento entre 2 e 3 diretor $=0.323309 \lambda$;

espaçamento entre fonte e refletor $=0.1924818 \lambda$.

Esses dados otimizados fornecem os parâmetros de diretividade, razão frente-costa e ângulo de meia potência apresentados abaixo: Ângulo de meia potência (plano E) $=40,86$ graus; Ângulo de meia potência (plano H) $=44,89$ graus; Razão frente-costa (plano E)=10,2482 dB; Razão frente-costa (plano H)=10,2372 dB; Diretividade $=12,170$ dB. Nota-se que o processo de otimização aplicado neste trabalho na síntese da antena Yagi-Uda de cinco elemento, obteve resultados significativos para a diretividade, para a razão frente-costa, tanto no plano E quanto no plano $\mathrm{H}$, e para o ângulo de meia potência. A Figura 3 mostra o diagrama de radiação para o plano $\mathrm{H}$ da antena otimizada.

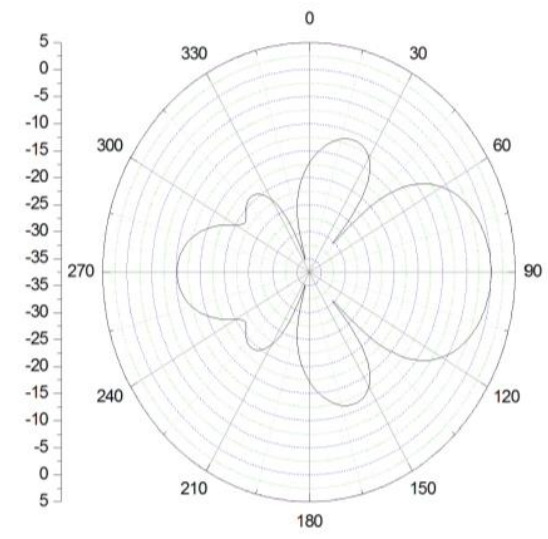

Figura 3: Diagrama de radiação (plano H) da antena Yagi-Uda de cinco elementos com os dados otimizados pelo método de Gauss-Newton.

\section{CONCLUSÃO}

O projeto de uma antena Yagi-Uda reunindo algumas características requeridas para este dispositivo foi desenvolvido usando a técnica de otimização de Gauss-Newton. Para tanto, foram especificados o número de elementos, sendo um refletor e um elemento energizado, diretividade, razão frente-costa e ângulo de meia. Os valores dos tamanhos e dos espaçamentos entre os elementos foram ajustados pelo processo de otimização.

Um projeto inicial da antena foi estabelecido e, a partir desses dados, aplicou-se o método de Gauss-Newton até que as faixas de valores das características pré-estabelecidas fossem atingidas.

A modelagem direta, ou seja, o projeto da antena Yagi-Uda a partir das entradas de comprimento e espaçamento entre os elementos da antena e como saída a diretividade, ângulo de meia potência e razão frente-costa, foi realizado a partir da aplicação do método dos momentos equação integral de Pocklington.

Este procedimento foi aplicado em antenas com cinco elementos, os resultados mostraram que o processo de otimização de Gauss-Newton apresenta-se como uma eficiente ferramenta para síntese de antenas Yagi-Uda. 


\section{REFERÊNCIAS}

[1] C. Balanis, “Antenna Theory and Design”. Jhon Wiley \& Sons, 1997, ISBN 0-471-59268-4.

[2] D. K. Cheng, "Gain optimization for Yagi-Uda array", IEEE Antennas and Propagation Magazine, vol. 33, pp. $42-45$, june 1991.

[3] E. A Jones and W. T. Joines, "Design of Yagi-Uda antennas using genetic algorithmis", IEEE Transactions and Propagation, vol. 45, pp. 1386 - 1392, setembro 1997.

[4] R. M. Ramos, R. R. Saldanha, R. H. C. Takahashi e F. J. S. Moreira,"Otimização multiobjeto aplicada ao projeto de antennas filamentares”, Ciência e Engenharia, SBMag/CBMag 2002 - Edição Especial, pp. 67- 70, dezembro 2003.

[5] V. J. da C. Farias, "Interpretação de dados de polarização induzida usando o modelo fractal para resistividades complexas e imagens tomográficas”, Programa de Pós-Graduação em Engenharia Elétrica, Centro Tecnológico, Universidade Federal do Pará, Tese de Doutorado, 151 p.

[6] J. J Xia, T. M. Habashy, and J. A Kong, "Profile inversion in a cylindrically stratified lossy medium". Radio Science, Vol. 29, pp. 1131 - 1141, 1994. [1] R. Courant, Variational methods for the solution of problems of equilibrium and vibrations, Bull. Amer. Math. Soc., 49 (1943) 1-23. 